\title{
ÜBER ELEKTROMOTORISCHE KRAFT UND CHEMISCHES GLEICHGEWICHT. NACH VERSUCHEN DES HERRN KNÜPFFER.
}

In seiner bekannten Abhandlung 1 ) in der schwedischen Akademie hat van't Hoff im Jahre I 886 auf energetischem Wege eine quantitative Beziehung zwischen der elektromotischen Kraft einer Reaktion und ihrer chemischen Gleichgewichtskonstante aufgestellt; welche man in Verbindung mit der Ionentheorie von Arrhenius als die Grundlage der modernen Lehre vom elektrolytischen Gleichgewicht betrachten kann. Diese Beziehung lautet:

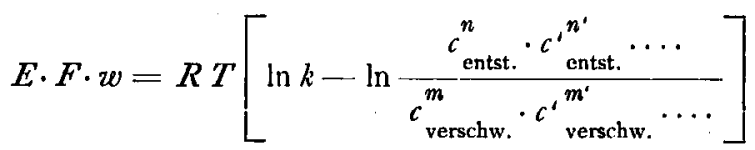

Hierin bedeuten:

$E$ die elektromotorische Kraft der Reaktion,

$F$ die Faraday'sche Konstante pro I $g$-Äquivalent,

$z$ die Wertigkeit der Reaktion,

$k$ die Gleichgewichtskonstante der Reaktion,

$c_{\text {entst. }}$ und $c_{\text {verschw. die angewandten Konzentra- }}$ tionen der bei der Reaktion entstehenden resp. verschwindenden Stoffe, wie sie von vornherein in der zu messenden Kette vorhanden sind,

$n$ und $m$ die Anzahl $\mathrm{g}$-Molen des zugehörigen Stoffes, welche bei Durchgang der Elektricitătsmenge $F \cdot w$ entstehen resp. verschwinden,

$R$ die Gaskonstante,

$T$ die absolute Temperatur.

$\mathrm{Zu}$ bemerken ist noch, dass die obige Gleichung nur für verdünnte Stoffe, also Gase und verdünnte Lösungen gültig. ist. Phasen von konstanter $\mathrm{Zu}$ sammensetzung (reine Metalle und feste Bodenkörper etc.) brauchen nicht mitgerechnet zu werden. Die Ionen sind als individuelle Stoffe $z \mathfrak{u}$ rechnen, wie Arrhenius, Ostwald, Nernst u. A. gezeigt haben. Für die Gaskette aus Sauerstoff und Wasserstoff ist übrigens die Gleichung in einem speziellen Falle implicite auch von Helmholtz ${ }^{2}$ ) benutzt worden, um die freiwillig aus reinem Wasser abgespaltene unmessbar kleine Menge von Knallgas vorauszuberechnen.

Wie man sieht, gestattet die obige Gleichung im zweiten Gliede der rechten Seite sofort, vorauszuberechnen, wie sich $E$ andert, wenn man in der

1) Svensk. Vetensk. Akad. Handlingar Bd. 21, Nr. 17. Lois de l'équilibre chim. S. 50. ván't Hoff-Cohen, Studien 2. chem. Dynamik, S. 274. Nernst, Lehrb. S. 56r. Ostwald, Z. phys. Chem. I5, S. 399.

2) Berliner Akad. I883. S. 660. Ges. Abhandl. III, S. I07.
Kette die Konzentration der bei Stromdurchgang verschwindenden und auftretenden Stoffe willkürlich andert. Nach dieser Seite hin hat die van 't Hoff sche Gleichung zahlreiche Bestätigung erfahren, vor allem in den wichtigen Arbeiten von $\mathrm{Nernst}^{3}$ ) für die Reaktion:

Metall $\rightleftarrows$ Metallionen

zum Beispiel

$$
Z_{\text {Metall }} \rightleftarrows Z_{n}++
$$

ebenso hat uns Herr Peters ${ }^{4}$ ) soeben für die Konzentrationsfunktion einen Beleg, gegeben in dem Falle $\quad \mathrm{Fe}^{+}+++\underset{\mathrm{Fe}}{+}+$

Das erste logarithmische Glied der rechten Seite der Fundamentalgleichung gestattet uns aber, aus rein chemischen Daten die elektromotorische Kraft (allgemein die freie Energie, s. Nernst, Lehrb. S. 5 I I) einer Reaktion bei bekannten Konzentrationen vorauszuberechnen, wenn wir ihre Gleichgewichtskonstante kennen, und umgekehrt aus der Messung der elektromotorischen Kraft einer umkehrbaren Zelle die chemischen Gleichgewichtsbedingungen der in ihr stattfindenden umkehrbaren Reaktion quantitativ vorauszubestimmen.

Eine experimentelle Prüfung dieser Anwendbarkeit der Gleichung ist noch verhältnismässig selten ausgeführt worden, aber wohl an einigen besonders wichtigen Fällen.

So hat zuerst Ostwald ${ }^{5}$ ) gezeigt, dass sich aus der Săurealkaligaskette für die Reaktion:

$$
\stackrel{+}{H}+\overline{O H} \rightleftarrows H_{2} O_{\mathrm{fl}}
$$

elektromotorisch berechnet $k \doteq \frac{\mathrm{I}}{\mathrm{I}, 4 \cdot \mathrm{IO}^{-14}}$,

wahrend Wys, Arrhenius und Kohlrausch ${ }^{6}$ ) u. a. auf verschiedenen analytischen Wegen (Verseifungsgeschwindigkeit, Hydrolyse und Leitfähigkeit) fanden

$$
k=\frac{\mathrm{I}}{\mathrm{I}, 3 \cdot \mathrm{IO}^{-14}} .
$$

Die zweite experimentelle Bestätigung rührt von Goodwin ${ }^{7}$ ) unter Ostwald's Leitung her und be-

8) Z. phys. Chem. 4, S. 147.

4) S. oben Vortrag von Herrn Dr. Peters.

5) Z. f. phys. Chem. II, S. 521 . Nernst, ibid. I4, S. 155 .

6) Z. phys. Chem. I 2, S. 522 ; I I, S. 827 ; I4, S. 330; 20, S. 283 .

7) Zeitschr. f. phys. Chem. I3, S. 64I. Ostwald, Lebrb. II, S. 879 . 
zieht sich auf den Lösungsvorgang, also zum Beispiel auf die Reaktion:

$$
A g C l_{\text {fest }} \rightleftarrows+\overrightarrow{A g}+\overrightarrow{C l} \text {. }
$$

Die Iöslichkeit $l$ des Chlorsilbers ist in diesem Falle gleich $\sqrt{k}$ und ergab sich aus der elektromotorischen Kraft einer Zelle, in der Chlorsilber sich auflöste, zu I,3 I $^{-5}$, während Kohlrausch und Rose auf einem anderen analytischen Wege fanden $l==\mathrm{I}, 4 \cdot \mathrm{IO}^{-5}$.

Es fehlte aber bisher noch ein Fall der doppelten chemischen Umsetzung, etwa wie bei Bariumsulfat mit Natriumcarbonat, oder der chemischen Verdrăngung.

So haben bereits Tammann und Nernst ${ }^{8}$ ) die fundamentale Bedeutung obiger Gleichung erkannt und sie an Fällen von der Art

$$
\mathrm{Zn}+\mathrm{H}_{2} \mathrm{SO}_{4} \rightleftarrows \mathrm{H}_{2}+\mathrm{ZnSO}_{4}
$$

zu prüfen versucht. $Z u$ völlig vergleichbaren quantitativen Resultaten sind sie aber nicht gelangt.

Ich selbst habe mich schon vor einigen Jahren mit dem Falle

$$
\mathrm{PbCl}_{2}+2 \mathrm{BrK} \longleftrightarrow \mathrm{PbBr}_{2}+2 \mathrm{ClK}
$$

beschaftigt, bin dabci aber auf Schwierigkeiten gestossen, die Herrn $\mathrm{K} n$ üpffer auch bei den analogen Thalliumsalzen begegnet sind und wahrscheinlich auf der Bildung von Mischkrystallen beruhen.

Es ist nun aber Herrn $\mathrm{Knüpffer}{ }^{9}$ ) auf meine Veranlassung gelungen, die obige Gleichung für den Fall der umkehrbaren doppelten chemischen Umsetzung:

$T l C l_{\text {fest }}+K S C N_{\text {Lïsung }} \leftrightarrows T l S C N_{\text {fest }}+K C l_{\text {Liisung }}$ zu prüfen. $\mathrm{Zu}$ diesem Zwecke schüttelte er Lösungen von Rhodankalium mit festem Chlorthallium und ebenso Lösungen von Chlorkalium mit festem Rhodanthallium, bis nach obiger Reaktion Gleichgewicht eingetreten war, und bestimmte dann analytisch das Verhaltnis von Chlor zu Rhodan in der Lösung, mithin die Gleichgewichtslionstante obiger Reaktion.

Ferner bestimmte Herr Knüpffer die elektromotorische Kraft von umkehrbaren Ketten nach dem Schema:

$T l_{\text {amalgan }}\left|T l C l_{\text {fest }} K C l_{\text {Lïsung }}\right| K S C N_{\text {Lïsung }} T l S C N_{\text {fest }} \mid T l_{\text {amalgam }}$

Er erhielt folgende Tabelle:

8) Zeitschr. f. phys. Chem. 9, S. I. Nernst, Lehrb. S. $56 \mathrm{I}$.

9) Die ausführliche Abbandlung wird bald in der Zeitschr. f. phys. Chem. erscheinen.

Gleichgewichtskonstante:

$k$ analytisch-

Temperatur: chemisch gefunden $k$ elektromot. berechnet

$\begin{array}{rrr}39,9^{\circ} & 0,85 & 0,88 \\ 20,0_{0} & \mathrm{I}, 24 & \mathrm{I}, 26 \\ 0,8^{\circ} & \mathrm{I}, 74 & \mathrm{I}, 79\end{array}$

Aus der van't Hoff'schen Gleichung, die sich hier bewährt, geht auch hervor, dass das, was chemisch im Gleichgewicht ist, es auch elektrolytisch ist, denn wird der zweite Logarithmus in der Klammer gleich dem ersten, so wird $E=$ o. Dieser Schluss ist für feste Phasen konstanter Zusammensetzung bereits von Ernst Cohen ${ }^{10}$ ) (Umwandlungselement), sowie von Luther ${ }^{11}$ ) (verschiedene Lösungsmittel gesättigt mit denselben Bodenkörpern) experimentell bestătigt worden. (Theoretisch, aber nicht experimentell hat auch Herr Bucherer unabhängig diesen letzten Fall aufgestellt.) In unserem Falle wird bei derjenigen Temperatur die Kette den Wert $E=$ o, also Polwechsel haben, bei welcher die Gleichgewichtskonstante den Wert des in der Zelle gerade willkürlich angewandten Konzentrations-Verhăltnisses $C l: S C N$ passiert, wenn also die in der Zelle angewandten Konzentrationen von Chlor und Rhodan bei dieser Temperatur auch im chemischen Gleichgewichte sind. Herrn Knüpffer's Versuche zeigen denn auch, dass man aus den in der Kette angewandten Konzentrationen und der Gleichgewichtskonstante die Temperatur des Polwechsels vorausberechnen kann:

Polwechseltemperatur: gef. ber.

Kette I ... $42,3^{\circ} \quad 41^{\circ}, 3^{\circ}$

Kette II ... 9, 9,2 ${ }^{\circ} \quad 7,6^{\circ}$

Oberhalb dieser Temperatur arbeitet die Kette in der endothermen Richtung der Reaktionsgleichung, unterhalb der Polwechseltemperatur mit der umgekehrten exothermen Reaktion.

Herr Knüpffer lat ferner die Wärmetönung $q$ seiner Reaktion gemessen und kann hieraus nach van't Hoff's ${ }^{12}$ ) bekannter Gleichung

$$
\ln \frac{k_{2}}{k_{1}}=\frac{q}{R}\left(\frac{\mathrm{I}}{T_{1}}-\frac{\mathrm{I}}{T_{2}}\right)
$$

das Verhältnis der $k$-Werte bei verschiedenen Temperaturen bestimmen, ebenso wie aus den bei verschiedenen Temperaturen gemessenen elektromoto-

10) Z. f. phys. Chem. 14, S. 53,535 ; 16, S. 453.

${ }^{11}$ ) Z. f. Chem. 19, 529. Z. f. phys. Chem. 20, S. 328.

$\left.{ }^{12}\right)$ l. c. Svensk. Vetensk. Akad. van't Hoff-Cohen, Studien. S. 152. Nernst, Lehrb. 509. 
rischen Kräften. Er erhielt so folgende 3 unabhängige Reihen:

Verhaltnis der Gleichgewichtskonstanten bei verschiedenen Temperaturen: aus der aus der direkt chemischWärme- elektromot. analytisch tönung ber. Kraft ber. bestimmt

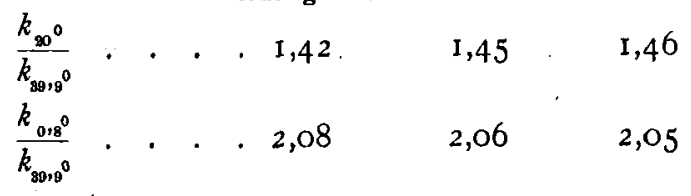

\section{ÜBER LÖSLICHES GOLD.}

Meine Herren! Herr Dr. Bredig hat uns gestern eine Reihe von interessanten Eigenschaften des elektrischen Lichtbogens vorgeführt. $\operatorname{Er}$ erhielt u. a. durch Zerstauben von Metallen unter Wasser dunkel gefarbte Flüssigkeiten, in denen die Metalle so fein zerteilt waren, dass man sie für gelöst.halten könnte; es sind das aber keine Lösungen, denn sie verlieren nach längerem Stehen zum Teil ihren Metallgehalt. Zerteilt man die Metalle noch weiter, so gelangt man zu Flüssigkeiten, die nicht mehr absetzen, zu colloidalen Lösungen von Metallen. Von solchen Lösungen waren bisher nur diejenigen des Silbers durch die Arbeiten von $\mathrm{Ca}$. Lea bekannt.

Es ist mir nun gelungen, wässerige Lösungen von Gold herzustellen. Sie sehen ganz so aus wie Goldrubinglas. Die Lösung, die ich hier habe, ist ausserordentlich verdünnt; sie enthält nur $5 / 1000 \%$ Gold. Sie ist aber trotzdem stark gefarbt. Wenn man diese Lösung der offenen Dialyse an einem warmen Orte unterwirft, so erhalt man eine konzentriertere Lösung, welche viel dunkler aussieht. Die vorliegende konzentrierte Lösung enthalt $1 / 10 \%$ Gold; sie ist aber schon sehr dunkel gefärbt und erscheint getrübt, weil sie übersättigt ist.

Die Herstellung dieser Flüssigkeiten ist sehr einfach, wenn die Vorschriften, die ich publizieren werde, genau befolgt werden. Man erhält wässerige Goldlösungen, wenn man sehr verdünnte Goldchloridlösungen schwach alkalisch macht und mit Formaldehyd behandelt. Konzentriert man die Flüssigkeiten im Dialysator, so bleibt das Gold gelöst und die Lösung kann auf diese Weise von den darin enthaltenen Salzen teilweise befreit werden. Wenn die Membran dicht schliesst, so geht das Gold nicht in das darunter befindliche Wasser. Das Gold ist also nicht fähig, die Membran zu durchdringen. Bei sehr weitgehender Konzentration schlagt
Der vorliegende Fall ist also ein besonders mannigfaltiges und anschauliches Beispiel für die Fundamentalbeziehungen des chemischen und elektrolytischen Gleichgewichts bei konstanter und bei variabler Temperatur.

Herrn Knüpffer sage ich an dieser Stelle noch besonders meinen Dank.

Eine Debatte findet nicht statt.

Herr Dr. Zsigmondy: sich das Gold als schwarzes Pulver auf der Membran nieder; nach dem Trocknen erscheint dieselbe dann glänzend, vergoldet, wie Sie an vorliegender Probe sehen können.

Eigenschaften: Wenn man die rote Lösung mit Kochsalz oder mit verdünnten Säuren versetzt, dann andert sich die Farbe; dieselbe wird momentan blau, im blaugefarbten Golde ist das Metall schon zu grösseren Teilchen vereinigt. Bewirkt man durch einen weiteren Zusatz von Salz, dass das Gold noch mehr zusammengeht, dann fällt es pulverförmig heraus. (Versuch!). Es wird bereits schwarzviolett. Dass das, was herausfällt, wirklich Gold ist, habe ich durch Analyse nachgewiesen.

Bei der Elektrolyse scheidet sich das Gold an der positiven Elektrode als schwarzes Pulver $a b$, das nach dem Trocknen Metallglanz annimmt. Trennt man die positive Elektrode von der Flüssigkeit durch eine Membran, so fallt das Gold auf dieser als blauschwarzes Pulver nieder. Hierin unterscheidet sich das colloidal gelöste von dem salzartig gelösten Golde.

Eine interessante Erscheinung ist die, dass. sich auf der Flüssigkeit Schimmelpilze bilden, wenn man sie offen stehen lässt. Die Schimmelpilze nehmen das Gold aus der Flüssigkeit. Ihr Mycelium sieht dann schwarz oder tief dunkelrot aus. Lässt man den Pilz auf Glas trocknen, so bleibt ein Goldfleck zurück, der unter dem Mikroskope das Aussehen eines goldglänzenden Gewebes annimmt.

Die Kraft des Pilzes, das Gold aufzunehmen, ist so gross, dass manchmal die Flüssigkeiten durch stark wuchernde Pilze ganz entfärbt worden waren. In diesem Fläschchen ist eine Lösung von colloidalem Zinnsăurehydrat enthalten; ich habe sie auf anderem Wege hergestellt als Graham die seine. 